\title{
REÚSO DE DISPOSITIVOS MÉDICOS DE USO ÚNICO E IMPLICAÇÕES PARA A SEGURANÇA DO PACIENTE
}

\author{
Reuse of single use medical devices and implications for patienty safety
}

Reutilización de dispositivos médicos de uso individual e implicaciones para la seguridad del paciente

Eliana Auxiliadora Magalhães Costa* ${ }^{1 *}$

RESUMO: Objetivo: Descrever aspectos do reúso dos dispositivos médicos de uso único e as implicações dessa prática para a segurança do paciente. Método: Ensaio acadêmico, utilizando dados de revisão integrativa e expertise da autora. Resultados: O reúso de produtos de uso único é realidade mundial e ocasiona debates regulatórios, técnicos, econômicos, éticos e de segurança do paciente, denotando diversos interesses dos distintos atores envolvidos: Estado, fabricantes, serviços de saúde, academia, profissionais e usuários. Embora haja risco teórico, dados não identificam relação causal entre evento adverso e reúso desses produtos. Existem argumentos a favor e contra que compreendem riscos e benefícios e justiça distributiva e social. O rótulo desses produtos representa nó crítico e elemento fomentador dos dilemas que permeiam essa prática. Conclusão: Há consenso de que o reúso de um produto médico deve ter o mesmo padrão de segurança, independentemente se rotulado como de uso único ou de multiuso. Alguns produtos ditos de uso único podem ser seguramente reusados, mas essa prática requer condições organoestruturais dos serviços de saúde, além de expertise, adoção de protocolos e supervisão dessas atividades. Palavras-chave: Dispositivos médicos. Exposição ao risco. Segurança do paciente.

ABSTRACT: Objective: to describe aspects of the reuse of single-use medical devices and implications for patient safety. Method: academic essay, using integrative review data and author's expertise. Results: the reuse of single-use products is a worldwide reality and causes regulatory, technical, economic, ethical and patient safety debates, denoting several interests of the different actors involved: State, manufacturers, health services, academia, professionals and users. Although there is a theoretical risk, data do not identify a causal relationship between adverse events and reuse of these products. There are arguments for and against and are involved: risks and benefits, distributive and social justice. The label of these products represents a critical node and fomenting element of the dilemmas that permeate this practice. Conclusion: There is consensus that the reuse of a medical product should have the same safety standard, regardless of whether labeled as single-use or multipurpose. Some so-called single-use products can be safely reused, but this practice requires organo-structural conditions of health services, as well as expertise, adoption of protocols and supervision of these activities. Keywords: Medical device. Risk-taking. Patient safety.

RESUMEN: Objetivo: Describir aspectos de la reutilización de dispositivos médicos de un solo uso e implicaciones para la seguridad del paciente. Método: ensayo académico, utilizando datos de revisión integradores y la experiencia del autor. Resultados: La reutilización de productos de un solo uso es una realidad mundial y provoca debates regulatorios, técnicos, económicos, éticos y de seguridad del paciente, que denotan diversos intereses de los diferentes actores involucrados: Estado, fabricantes, servicios de salud, academia, profesionales y usuarios. Aunque existe un riesgo teórico, los datos no identifican una relación causal entre el evento adverso y la reutilización de estos productos. Hay argumentos a favor y en contra y están involucrados: riesgos y beneficios, justicia distributiva y social. La etiqueta de estos productos representa un nodo crítico y un elemento que fomenta los dilemas que impregnan esta práctica. Conclusión: Existe un consenso de que la reutilización de un producto médico debe tener el mismo estándar de seguridad, independientemente de si está etiquetado como de uso único o multipropósito. Algunos de los llamados productos de un solo uso pueden reutilizarse de manera segura, pero esta práctica requiere condiciones organoestructurales para los servicios de salud, además de experiencia, adopción de protocolos y supervisión de estas actividades. Palabras clave: Dispositivos médicos. Asunción de riesgos. Seguridad del paciente.

'Doutora em Saúde Pública. Professora Adjunta da Universidade do Estado da Bahia - Salvador (BA), Brasil.

*Autora correspondente: costaeliana2003@hotmail.com

Recebido: 06/16/2019 - Aprovado: 03/04/2020

https://doi.org/10.5327/Z1414-4425202000040009 


\section{INTRODUÇÃ̃O}

Os dispositivos médicos representam parcela significativa das tecnologias duras utilizadas nos serviços de saúde e são usados para diagnosticar, tratar ou prevenir doenças. Esses dispositivos, largamente utilizados em todos os ramos da saúde, são definidos pelos fabricantes como artigos reusáveis ou de uso único. Os reusáveis são considerados bens duráveis, sua reutilização requer a ação do reprocessamento, que é processo de muitas etapas que consiste em converter um produto contaminado em um dispositivo pronto para uso ${ }^{1}$.

Os produtos de uso único são designados para serem usados somente uma vez, em um único paciente. Esses produtos surgiram com o advento da indústria dos plásticos e ganharam popularidade em função, dentre outros motivos, do crescimento da infecção causada pelo vírus da imunodeficiência humana. Como resultado, muitos produtos de assistência à saúde, que eram inicialmente fabricados como reusáveis, passaram a ser fabricados com polímeros plásticos baratos, sendo descartáveis após uso único, o que acarretou grande benefício econômico para os fabricantes ${ }^{1}$.

Independentemente do rótulo do fabricante, a prática do reúso de produtos de uso único é realidade mundial, que foi iniciada na década de 1970, e desde então há relatos de reúso desses produtos em vários países do mundo, mesmo em nações desenvolvidas, sobretudo naquelas onde o reprocessamento é proibido $^{2,3}$. Essa tendência tem intensificado vários debates e considerações sobre segurança do paciente, consentimento informado, questões técnicas, econômicas, ambientais, legais e éticas e aspectos regulatórios para fabricantes e reprocessadores, denotando interesses distintos por parte dos atores políticos envolvidos: Estado, fabricantes de produtos, serviços de saúde, academia, profissionais de saúde, associações de classe e usuários ${ }^{4-9}$.

São muitos os argumentos que advogam a favor e contra o reúso de produtos de uso único ${ }^{1,4}$. Os favoráveis justificam os impactos positivos nos custos e no meio ambiente, pois reduzem o volume de resíduos gerados oriundos dos cuidados à saúde. Os críticos do reúso argumentam que esses produtos não são designados para múltiplos usos e que a reutilização pode trazer risco de transmissão de infecção e de endotoxinas, inconfiabilidade funcional, quebra da integridade do produto ou bioincompatibilidade ${ }^{410}$.

Embora o reprocessamento e o reúso de produtos de uso único possua um risco teórico à saúde, evidências clínicas apresentam que certos produtos podem ser seguramente reprocessados. Todavia, isso não significa que o reprocessamento desses produtos é sempre seguro ${ }^{11,12}$.
Nesse sentido, este estudo procura responder à seguinte questão norteadora: O reúso de dispositivos médicos de uso único atenta contra a segurança do paciente usuário desses produtos?

\section{OBJETIVO}

Descrever aspectos históricos e atuais acerca do reúso de produtos de uso único e as implicações dessa prática para a segurança do paciente usuário desses materiais, de modo a contribuir para análise das questões emblemáticas relacionadas à reutilização desses dispositivos.

\section{MÉTODO}

Trata-se de ensaio acadêmico em que se utilizam dados de revisão integrativa da literatura, método que permite a síntese de resultados de estudos com distintas metodologias, sem ferir a filiação epistemológica desses resultados. O estudo é composto de cinco etapas: formulação do problema, coleta de dados, análise de dados, síntese e divulgação dos resultados ${ }^{13}$.

Os estudos foram obtidos por meio do portal da Biblioteca Virtual em Saúde (BVS), o qual inclui busca nas bases Literatura Latino-Americana e do Caribe em Ciências da Saúde (LILACS), Índice Bibliográfico Español en Ciencias de la Salud (IBECS), National Library of Medicine/NLM (MEDLINE), The Cochrane Library, Scientific Eletronic Library Online (SciELO), National Library of Medicine/ NLM (PubMed) e Web of Science.

Os descritores da saúde utilizados foram: reprocessing single use medical device, reuse single use medical device, risk of reuse single use medical device, risk of reuse single use medical device, com auxílio do operador booleano AND.

Os critérios de inclusão foram: artigos em inglês, espanhol e português, que abordaram os temas risco, reprocessamento e reúso de produtos de uso único, sem restrição de tempo de publicação. Foram excluídos artigos de reúso e reprocessamento de produtos em serviços de hemodiálise e em serviços odontológicos e os publicados em outros idiomas.

A busca dos dados foi feita online, de outubro a dezembro de 2017, sendo obtidos 870 artigos. Após leitura do título e do resumo, segundo os critérios definidos, foram excluídos 827 artigos e selecionados 20 , entre os quais alguns foram citados nas referências dos artigos selecionados, perfazendo o total de estudos que integram esse estudo. 
Após a seleção, os artigos foram lidos e analisados utilizando-se instrumento de coleta de dados que incluiu: nomes dos autores, título do artigo, referência, objetivos, material e método, resultados e conclusão.

Neste estudo, utiliza-se o termo dispositivo médico como sinônimo de produto para saúde, equipamento, material e artigo médico, em conformidade com a Agência Nacional de Vigilância Sanitária do Brasil (ANVISA). Usam-se também como sinônimos os termos reprocessamento ou processamento de produtos, a despeito de considerações sobre as diferenças entre esses.

\section{RESULTADOS E DISCUSSÃO}

\section{Reúso de produtos de uso único e implicações para a segurança do paciente}

A prática do reprocessamento e reúso de produtos de uso único tem sido essencialmente estudada do ponto de vista ético ("deveria ser feito?") e do ponto de vista técnico ("como deveria ser feito?”) ${ }^{3}$. Nesse sentido, existe amplo número de publicações sugerindo segurança e eficácia no reúso de produtos descartáveis, sobretudo na área dos procedimentos de intervenção cardíaca, entretanto esses estudos variam em metodologia e qualidade, o que dificulta o consenso sobre o reúso desses produtos. Além disso, informações sobre eventos adversos relacionados com produtos médicos são frequentemente voluntárias e, por conseguinte, são sub-reportadas, podendo não representar a totalidade dos $\operatorname{casos}^{2,5,8,11,12,14}$.

Em 1990, a Food and Drug Administration (FDA), dos Estados Unidos da América (EUA), empreendeu estudo acerca da segurança do reúso de produtos de uso único e concluiu que o padrão de eventos adversos de pacientes expostos ao reúso desses produtos independe de serem de uso único ou de uso múltiplo ${ }^{2,5,11}$. Em 2008, o United Status Government Accountability Office (GAO) afirmou não haver relação causal entre injúrias e mortes de pacientes e reúso de produtos de uso único ${ }^{11}$.

Desse modo, não existem dados suficientes, nem do FDA, nem de outros estudos, sobre a segurança de produtos de uso único reprocessados, quando comparados com produtos originais ${ }^{8,11,15,16}$. Para tal comparação, seria necessário identificar os tipos de produtos e de eventos adversos, o número do produto de uso único original dado pelo fabricante e do serviço quando é reprocessado, o número de vezes que cada produto de uso único foi reusado e a taxa de evento adverso associado com o produto original ${ }^{11}$. O FDA tem analisado dados de eventos adversos relacionados a produtos de uso único reprocessados e não tem identificado associação causal entre o evento adverso e o fato do produto de uso único ser reprocessado ${ }^{11}$.

Alguns organismos internacionais têm-se posicionado acerca do reúso de produtos de uso único.

O Center for Disease Control and Prevention (CDC) dos EUA posiciona-se favoravelmente pelo reúso de produtos de uso único e afirma que esses produtos não possuem risco, se puderem ser adequadamente limpos e esterilizados 5 .

A European Medical Technology Industry Association posiciona-se contrária ao reúso de produtos de uso único e afirma que a segurança do paciente é ameaçada quando esses produtos são reusados, em função de risco de transmissão de infecção cruzada, inabilidade da limpeza desses dispositivos, presença de resíduos, alteração dos componentes dos materiais, falência mecânica, entre outros argumentos ${ }^{15-17}$.

$\mathrm{Na}$ comunidade europeia, a European Association of Medical Devices Reprocessors (EAMDR) conclamou os estados membros para analisarem como as regulações europeias estão implementando o reúso desses produtos e afirma que a "alta qualidade do reprocessamento de produtos em todos os estados membros somente pode ser garantida se realizada independente do rótulo escolhido pelo fabricante"15-18, declaração que prioriza a qualidade do processo, independentemente do produto, de uso único ou não.

Para a Joint Commission International (JCI), se um hospital decide reusar produtos de uso único deve avaliar criticamente as condições do departamento de limpeza, desinfecção e esterilização dos produtos, bem como procedimentos e pessoal ${ }^{8}$.

A Organização Mundial da Saúde (OMS) declara que o reúso de produtos de uso único requer políticas escritas e que produtos críticos e semicríticos só devem ser reusados por reprocessador licenciado ${ }^{8}$.

Para expertos, como os professores Axel Kramer e Marc Kraft, do departamento de tecnologia médica de Berlim, o "critério crucial é que exista um procedimento validado para o reprocessamento de um produto médico. Se o produto é de multiuso ou de uso único, é irrelevante". Para Marc Kraft, a "validação do procedimento do reprocessamento tende a excluir um aumento do risco". Nesse caso, não há ameaças higiênicas, nem técnico-funcionais ${ }^{5}$.

A Federação Internacional de Controle de Infecção considera que cinco questões devem ser respondidas positivamente pelos reprocessadores para que o reúso de produtos descartáveis seja considerado seguro:

- O produto mantém-se íntegro e funcional?

- É passível de limpeza? 
- Pode ser esterilizado?

- O reúso é custo-efetivo?

- Quem será responsável caso ocorra um evento adverso?8,19

É recomendado que o produto de uso único não seja reusado e reprocessado se:

- não pode ser adequadamente limpo;

- a esterilidade do produto reprocessado não pode ser seguramente demonstrada;

- a integridade, a funcionalidade e a segurança do produto de uso único reprocessado difere do produto original.

A segurança e a efetividade do reprocessamento de produtos de uso único devem ser conduzidas segundo processos padronizados e monitorados, com a mesma garantia de qualidade dos produtos originais ${ }^{2,5-6,8,16}$.

Existem três práticas acerca de produtos de uso único que envolvem discussões sobre a reutilização e distintas condutas adotadas por muitos serviços:

- produtos de uso único abertos, mas não usados;

- produtos de uso único colocados numa mesa cirúrgica, mas não usados;

- produtos de uso único abertos e usados num paciente.

Autores discutem que produtos de uso único abertos e não utilizados deveriam ser elegíveis para reúso sem discussões. Outros defendem que cada prática requer consideração cuidados $\mathrm{a}^{20}$.

O número de vezes que o dispositivo de uso único pode ser reusado é também uma situação desafiadora para a segurança do paciente. Autores afirmam que o número máximo de reúso sustentável de um produto descartável é parâmetro fundamental e deve ser avaliado por meio de análise física, química, microbiológica e testes funcionais ${ }^{2,12}$. Essa situação pode ser aplicada igualmente para os produtos de uso múltiplo, uma vez que esses também não podem ser reprocessados e reusados de modo indeterminado ${ }^{20}$.

Em relação ao rótulo, a declaração de que o produto é de uso único ou de multiuso é baseada somente na decisão do fabricante, que qualifica um produto como de uso único por duas razões: porque acredita que o produto não é seguro nem confiável para ser usado mais de uma vez, ou porque o fabricante escolhe não conduzir estudos necessários para demonstrar que o produto pode ser rotulado como reusável. Nesse sentido, há ausência de considerações consistentes a respeito da definição de uso único pelo fabricante $e^{2,4,5,8,11,16}$.

Quando um produto médico é registrado como de uso único, isso somente significa que pode ser usado de maneira segura e confiável uma vez, contudo não implica que não pode ser usado de modo seguro mais de uma vez, se apropriadamente reprocessado. É importante ressaltar que fabricantes frequentemente mudam rótulos de produtos reusáveis para uso único, algumas vezes sem qualquer mudança significativa de desenho, desempenho ou material que poderia impossibilitar o reúso seguro ${ }^{15}$.

Ademais, produtos podem ser construídos de modo similar e classificados diferentemente por alternativa dos fabricantes que se beneficiam com o rótulo de uso único, uma vez que produtos assim definidos não requerem o mesmo grau de documentação e de validação para seu registro nos órgãos reguladores, ao contrário de produtos classificados como de uso múltiplo. Além disso, os órgãos reguladores não solicitam que os fabricantes apresentem provas de que o produto de uso único não pode ser reprocessado e de que o reúso possa ser inapropriado ou perigoso $\mathrm{O}^{2,4,11,13,15,19}$.

Na prática, a maioria dos produtos de uso único é tecnologicamente reusável. Estima-se que de 10 a $20 \%$ dos produtos classificados pelo fabricante como de uso único possam ser reprocessados por um número limitado de vezes ${ }^{16}$. Pesquisadores em posição aos fabricantes defendem que muitos produtos de uso único podem ser seguramente reprocessados e reutilizados nos hospitais ${ }^{4,5,16,20}$.

A estipulação de que produtos de uso único não podem ser reusados coloca os sistemas de saúde e a sociedade em posição de reféns financeiros do que dizem os fabricantes ${ }^{2}$. Alguns autores estão desafiando as premissas de que produtos de uso único são estritamente de uso único, não apenas por considerações financeiras, mas também ambientais ${ }^{2,20}$.

No Brasil, estudo realizado pela Sociedade Brasileira de Arritmias Cardíacas acerca dos rótulos de produtos de uso único registrados pelos fabricantes na ANVISA identificou que, dos 121 produtos médicos utilizados em procedimentos de eletrofisiologia registrados como de uso único, 86 $(71,7 \%)$ rótulos estavam em conformidade com a normativa em vigor e 34 (28,9\%) em desacordo. Os autores concluíram que as inconsistências nos rótulos desses produtos podem gerar erros de interpretação e decisões impróprias em relação ao seu uso ${ }^{21}$.

Outras questões complexas também são importantes e merecedoras de discussão em relação ao reúso de produtos de uso único:

- A questão do consentimento informado, que diz respeito à autonomia do paciente em escolher o que é melhor para ele; 
- A responsabilidade fiscal em promover o descarte de um produto que pode ser seguramente reusado;

- O comportamento ético em relação ao ambiente e às comunidades nas quais vivemos.

Impõe-se o questionamento: Estamos comportando-nos de maneira ética em relação ao ambiente e às comunidades onde vivemos, ao promover uso único de um produto que pode ser reusado ${ }^{14,20}$ ?

A análise dessas questões pode ser feita à luz da teoria do principialismo, ou teoria dos quatro princípios, que muito tem contribuído para propiciar soluções nos planos da ética individual e coletiva: beneficência (dever de promover o bem e agir para o melhor interesse para o paciente e para a coletividade), não maleficência (dever de não causar dano ao paciente), autonomia (dever de respeitar a vontade do sujeito) e justiça (princípio que valoriza adequada alocação dos recursos e necessidade de decidir o que e quem priorizar no acesso aos bens considerados finitos e escassos). Entretanto, esses princípios, apesar de claros e acessíveis, também apresentam dificuldade na sua operacionalização quando da questão do reúso de produtos ditos descartáveis: Qual paciente deverá receber um produto de uso único que nunca foi usado e qual deverá receber um produto reusado e reprocessado? Quem é o responsável pela decisão? O paciente tem condições de escolher ou deverá ser informado ${ }^{4,16}$ ?

As responsabilidades legais são óbvias e a instituição de saúde deve assumir a responsabilidade para o reuso de produtos de uso único, uma vez que contraria a orientação do fabricante, quando esse ao rotular o produto como de uso único, apenas se responsabiliza pela qualidade e eficácia do produto somente segundo sua recomendação ${ }^{8,14,16}$.

Outro aspecto do reúso desses produtos diz respeito à justiça distributiva e social, que requer a distribuição de risco e benefício para todos os pacientes ${ }^{12}$. A literatura reporta muitos riscos relacionados também ao reúso de produtos de multiuso e, nesse sentido, o foco apenas no reúso de produtos descartáveisdesvia a atenção para o processo de descontaminação dos produtos ditos reusáveis, que, a priori, requerem os mesmos padrões de qualidade e segurança. Nesse sentido, o reúso seguro de produtos de uso único, com o objetivo de melhorar o acesso à saúde, parece ser justificado eticamente, como tentativa de criar condições de igualdade de oportunidade e acesso à saúde e ao bem-estar.

\section{Síntese dos resultados}

Este estudo aponta várias questões emblemáticas relacionadas ao reúso de dispositivos médicos, tanto do ponto de vista técnico-operacional como de natureza ética, legal, jurídica e ambiental.

Os dados publicados questionam esse reúso, com base em considerações técnicas. Vários estudos sugerem segurança e eficácia no reúso para muitos produtos rotulados como de uso único e ausência de associação causal entre dano ao paciente e o fato do produto de uso único ser reprocessado ${ }^{11}$, entretanto é também claro que alguns produtos de uso único não são seguros para reprocessamento e reúso, dada a impossibilidade de limpeza e de esterilização, condição também aplicada ao reúso de produtos classificados como reusáveis.

Nesse sentido, a decisão sobre usar um produto, independentemente do rótulo de uso único ou múltiplo, requer processos padronizados, validados e monitorados para a garantia da qualidade e a minimização de riscos para os pacientes usuários de produtos reprocessados.

Para além das questões propostas, o rótulo dos produtos de uso único representa nó crítico e elemento fomentador dos dilemas que permeiam o reúso desses produtos.

A ausência de estudos no ato do registro que comprovem que um produto registrado como de uso único não pode ser reprocessado e que o reúso pode ser inapropriado ou perigoso para o paciente, não apenas torna inconsistente a definição de uso único, como cria situação de conflito para os órgãos reguladores e os serviços de saúde que se subordinam às declarações dos fabricantes.

Nesse sentido, desmitificar o rótulo desses produtos é crucial para a tomada de decisão regulatória e seus desdobramentos. Os produtos registrados como de uso único são de fato inseguros para a reutilização ou o fabricante tem outros motivos para esse rótulo?

Essa questão é o ponto chave do reúso de produtos médicos. A política regulatória deveria focar os sistemas de normatização nos processos desenvolvidos para a reutilização de um dispositivo de assistência à saúde, não submetendo a classificação do produto ao fabricante, tendo em vista que, mesmo os produtos classificados como reusáveis, não podem ser reutilizados de modo continuo, a despeito dessa indicação.

\section{CONSIDERAÇÕES FINAIS}

O reúso de produtos designados como de uso único envolve muitas questões, a começar pela dubiedade do próprio rótulo.

Há motivos para que os fabricantes optem por essa alternativa, e estudos mostram que o reúso desses produtos, quando realizado de maneira apropriada, pode ser seguro 
para o paciente, possibilitando tratamento de saúde efetivo e redução de custos no tocante aos produtos médicos.

Ademais, é importante considerar a ética em relação ao ambiente - ética da terra - e, nessa profusão crescente de descarte de resíduos, o reúso de produtos de uso único é considerado uma das melhores práticas de proteção ambiental.
O tema permanece como campo aberto a investigações sobre questões de natureza técnica, ética, econômica, ambiental e regulatória. Também se faz necessário compreender que alguns produtos ditos de uso único podem ser reusados, mas essa prática requer condições organoestruturais dos serviços de saúde, além de expertise, adoção de protocolos e supervisão de cada uma das etapas do reprocessamento.

\section{REFERÊNCIAS}

1. Kraft M. Framework conditions and requeriments to ensure the technical functional safety of reprocessed medical devices. GMS Krankenhaushyg Interdiszip. 2008;3(3).

2. Crawford TC, Eagle KA. Reuse of catheters and devices labeled for single use: evidence recommendations and oversights. Heart Asia. 2018;10(2):e011033. https://doi.org/10.1136/heartasia-2018-011033

3. Sloan T. First, do not harm? A framework for evaluation new versus reprocessed medical devices. J Oper Res Soc. 2010;61(2):191-201. https://doi.org/10.1057/jors.2008.137

4. Ponchon T, Pioche M. Reprocessing single-use devices: a new season in a long-running show? A European perspective. Endoscopy. 2017;49(12):1195-7. https://doi.org/10.1055/s-0043-121988

5. Kapoor A, Vora A, Nataraj G, Mishra S, Kerka P, Manjunath CN. Guidance on reuse of cardio-vascular catheters and devices in India: a consensus document. Indian Heart J. 2017;69(3):357-63. https:// doi.org/10.1016/j.ihj.2017.04.003

6. Association of periOperative Registered Nurses. Guidance statement: reuse of single use devices. Denver: AORN; 2012.

7. Kuniyoshi RR, Sternick EB, Nadalin E, Hachul DT. Reprocessing of medical products in electrophysiology. Arq Bras Cardiol. 2017;108(2):169-72. https://dx.doi.org/10.5935\%2Fabc.20170010

8. Mansur JM. Reuse of single use devices. Understanding risks and strategies for decision-making for healthcare organizations [Internet]. Joint Commission International; 2017 [acessado em 26 dez. 2018]. Disponivel em: http://www.jointcommissioninternational.org

9. Kwakye G, Brat GA, Makary MA. Green surgical practices for health care. Arch Surg. 2011;146(2):131-6. https://doi.org/10.1001/ archsurg. 2010.343

10. Costa EAM. Panorama internacional do reprocessamento de produtos para saúde de uso único. Rev SOBECC. 2016;21(4):203-9. https://doi. org/10.5327/Z1414-4425201600040005

11. United States Government Accountability Office. Reprocessed singleuse medical devices. FDA Oversight has increased and available information does not indicate that use presents and elevated health risk. Estados Unidos: United States Government Accountability Office; 2008.
12. Tessarolo F, Disertori M, Guarrera CGM, Favaretti C, Nollo G. Health technology assessment on reprocessing single-use catheters for cardiac electrophysiology: results of a three years study. In: Anais do 29th Annual International Conference of the IEEEEMBS, Lyon, France, 2007. Lyon; 2007.

13. Soares CB, Hoga LAK, Peduzzi M, Sangaleti C, Yonekura T, Silva DRAD. Revisão integrativa: conceitos, métodos utilizados na enfermagem. Rev Esc Enferm USP. 2014;48(2):335-45. https://doi.org/10.1590/ S0080-6234201400002000020

14. Unger S, Landis A. Assessing the environmental, human health, and economic impacts of reprocessed medical devices in a Phoenix hospital's supply chain. J Clean Prod. 2016;112:1995-2003. https:// doi.org/10.1016/j.jclepro.2015.07.144

15. The European Medical Technology Industry Association. Eucomed white paper on the reuse of single use devices [Internet]. The European Medical Technology Industry Association; 2009 [acessado em 31 dez. 2018]. Disponível em: http://www.medtecheurope.org

16. Mues AC, Haramis G, Casazza C, Okhunov Z, Badani KK, Landman J. Prospective randomized single-blinded in vitro and ex vivo evaluation of new and reprocessed laparoscopic trocars. J Am Coll Surg. 2010; 211 (6):738-43. https://doi.org/10.1016/j.jamcollsurg.2010.08.003

17. Jacobs P, Akpinar I. Single-use medical devices: economics issues. Heart Asia. 2018;10(2):e011034. https://doi.org/10.1136/ heartasia-2018-011034

18. Kwakye G, Brat GA, Makary MA. Green surgical practices for health care. Arch Surg. 2011;146(2):131-6. https://doi.org/10.1001/ archsurg.2010.343

19. Popp W, Rasslan O, Unahalekhaka A, Brenner P, Fischnaller E, Fathy $\mathrm{M}$, et al. What is the use? An international look at reuse of single use medical devices. Int J Hyg Environ Health. 2010;213(4):302-7. https://doi.org/10.1016/j.ijheh.2010.04.003

20. Moszczynzki A. Is once always enough? Revisiting the single use item. J Med Ethics. 2009;35(2):87-90. https://doi.org/10.1136/ jme.2008.025643

21. Kuniyoshi RR, Sternick EB, Nadalin E, Hachul DT. Reprocessing of medical products in electrophysiology. Arq Bras Cardiol. 2017;108(2):169-72. https://doi.org/10.5935/abc.20170010 